\title{
Nonlinear optical tagging diagnostic for the measurement of Fokker-Planck diffusion and electric fields
}

\author{
N. Claire, ${ }^{\text {a) }}$ M. Dindelegan, and G. Bachet \\ Equipe Turbulence Plasma, LPIIM, CNRS-Université de Provence, 13397 Marseille Cedex 20, France \\ F. Skiff \\ Department of Physics and Astronomy, University of Iowa, Iowa City, Iowa 52242
}

(Received 5 June 2001; accepted for publication 25 September 2001)

\begin{abstract}
Strong optical pumping of ions can result after a few microseconds of resonance excitation in a laser beam. However, both Fokker-Planck diffusion and acceleration due to macroscopic electric fields can remove an ion from resonance by changing the ion velocity on a similar timescale. Therefore, the time dependence of laser induced fluorescence can be influenced by particle acceleration and velocity-space diffusion. This effect which has already been used to measured Fokker-Planck diffusion, is extended to include the influence of an electric field and used to measure the electric field associated with an electrostatic shock in a multipolar gas discharge. (C) 2001 American Institute of Physics. [DOI: 10.1063/1.1419221]
\end{abstract}

\section{INTRODUCTION}

Velocity-space diffusion and electric mean fields are basic physical phenomena that determine transport phenomena in turbulent plasma. One way to explore experimentally the role of these phenomena in transport is through the use of test particles. The test particle velocity-space diffusion coefficient $D$ can be measured by "optical tagging." ${ }^{1}$ In this technique, one laser beam "tags" a given class of ion velocity and another beam is used to observe the diffusion of the tagged ions by laser induced fluorescence (LIF). ${ }^{2}$ However, if one analyzes the time dependent decay of the fluorescence light in a three level system like the one sketched in Fig. 1 for Ar II, the velocity diffusion coefficient can be obtained with only one laser beam using nonlinear optical tagging. ${ }^{3,4}$

The purpose of this article is to show that this nonlinear optical tagging can be extended to measure both electric field $E$ and velocity-space diffusion coefficient $D$ in the presence of a density perturbation associated with the propagation of an electrostatic shock.

Section II of the article is devoted to the parametric model, Sec. III to the experimental setup, Sec. IV to experimental results, and finally a discussion is in Sec. V

\section{THEORETICAL MODEL}

The essence of nonlinear optical tagging is the fact that laser induced fluorescence is nonlinear due to optical pumping. The LIF signal is proportional to the target (lower) state density, but even at laser intensity levels well below saturation (saturation being an intensity where the induced transition rate becomes comparable to the spontaneous transition rate) the target state density will be time dependent due to optical pumping by the laser light. For an ion with probabil-

\footnotetext{
a) Author to whom correspondence should be addressed; electronic mail: nclaire@up.univ-mrs.fr
}

ity $p(t)$ of being in the target state that experiences a laser induced transition rate $W(t)$, the probability $p$, derived from rate equations without collision terms, will decay according to

$$
\frac{d p}{d t}=-W(t)\left(1-\frac{A_{21}}{A_{T}}\right) p
$$

Here $A_{21}$ is the rate of spontaneous decay from the excited state back to the initial target state and $A_{T}$ is the total spontaneous decay rate of the upper state (see Table I). Equation (1) assumes that the laser intensity is well below saturation $\left(W \ll A_{T}\right.$ ). In a two level system, there will be no pumping, but in a three (or higher) level system such as shown in Fig. 1 there will be a decay of the target state probability. Because a real laser beam has a specific spatial and spectral intensity profile, the time-dependent position and velocity of an ion will affect the induced transition rate through changes in the laser intensity and the Doppler shift experienced by the particle along its orbit. Previously, nonlinear optical tagging has been analyzed through solving a system of coupled Eulerian kinetic equations where there is one kinetic equation for each quantum state of the ion. ${ }^{5}$ Here we exploit the separation of the classical mechanics of the ion orbit and the quantum mechanics of the state densities by using a Lagrangian approach. As long as the effects of photon momentum on the particle orbit (e.g., laser cooling) are negligible, then the Lagrangian approach offers a considerable computational advantage.

A Lagrangian approach begins with a description of the ion orbits in the plasma. Based on the nature of the orbits, the contribution of each ion to the LIF signal can be computed by calculating the effects of optical pumping along the specific orbit taken by the particle. This can be done by solving the rate equations that govern optical pumping with $W(x(t), v(t), t)$ being determined by the laser beam parameters together with the particle orbit $x(t), v(t)$. The total LIF signal is computed by summing over each kind of particle 


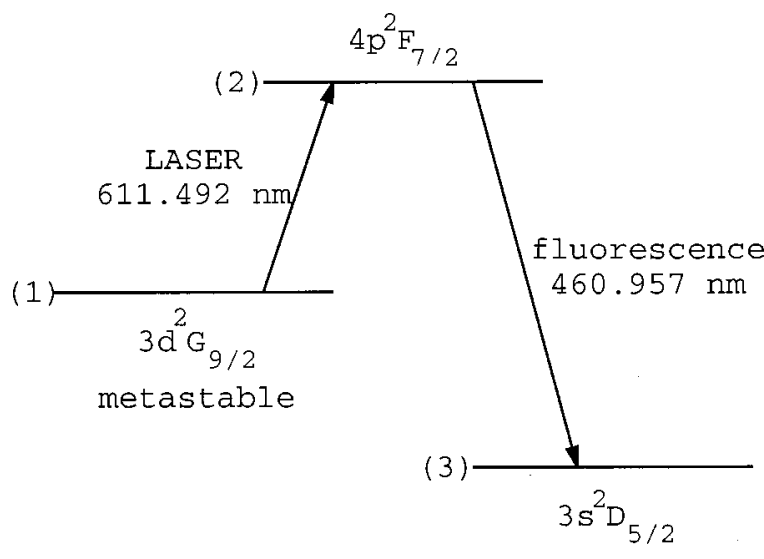

FIG. 1. Energy level diagram for LIF in Ar II.

orbit with a weighting according to the population of each kind of orbit. The equilibrium state densities of ions in a plasma are generally controlled by many processes including electronic excitation and quenching. Therefore, the use of nonlinear optical tagging to probe the nature of ion orbits, and thus infer an electric mean field and the velocity space diffusion coefficient, is greatly simplified by considering short optical pulses. Short in this context means short enough that all processes slower than optical pumping can be ignored. In the experiments considered here this implies $\delta t$ $<10^{-5}$ s. In fact the interesting dynamics will occur in a couple of microseconds.

To describe the ion orbits over a short time interval, but including the effects of diffusion, we employ a conditional probability function $P\left(x, v, t ; x^{\prime}, v^{\prime}, t^{\prime}\right)$ that specifies the probability of finding a particle at position $x$ and velocity $v$ at time $t$ given that the particle was at position $x^{\prime}$ and velocity $v^{\prime}$ at time $t^{\prime}$. This function is the Greens function of the kinetic equation for the ions. Here we will make a couple of additional simplifications that are useful for the experiment. We will consider laser beams that have a large spatial extent compared to the displacement of an ion during the light pulse and consider electric fields that have a small variation over the ion displacements and time interval. One consequence of these assumptions is that all ions at the same location $x^{\prime}$ will experience the same acceleration. The position of the measurement $x^{\prime}$ is retained as a parameter, but the coordinate $x$ will not enter into the kinetic equation. Because the Coulomb collision frequency is low, but the laser interacts with a narrow range of particle velocities, the velocity space diffusion term is important but the drag term is not important in the ion dynamics. It is possible to relax these assumptions, but the analysis becomes unnecessarily complicated for the present purposes. With these assumptions we can remove the spatial dependence of the kinetic equation and consider con-

TABLE I. Parameters of the theoretical model. The value of $r$ is known only to within an order of magnitude.

\begin{tabular}{cc}
\hline \hline Parameter & Value \\
\hline$A_{T}$ & $91.1 \times 10^{7} \mathrm{~s}^{-1}$ \\
$B$ & $1.55 \times 10^{20} \mathrm{~m} \times \mathrm{s}^{2} \times \mathrm{kg}^{-1}$ \\
$r$ & $\simeq 10^{4} \mathrm{~s}^{-1}$ \\
\hline \hline
\end{tabular}

ditional probability functions described by the equation:

$$
\frac{\partial P}{\partial t}+a\left(x^{\prime}, t^{\prime}\right) \frac{\partial P}{\partial v}=\frac{\partial}{\partial v}\left(D \frac{\partial P}{\partial v}\right) .
$$

Here $a\left(x^{\prime}, t^{\prime}\right)=e E\left(x^{\prime}, t^{\prime}\right) / m_{i}$ is the acceleration of ions due to an electric mean field $E$, and $D$ is an averaged velocity space diffusion coefficient. The equation is subject to the initial condition

$$
P\left(v, t^{\prime} ; v^{\prime}, t^{\prime}\right)=\delta\left(v-v^{\prime}\right) .
$$

Because of the Doppler effect, the function $P$ of interest is only sensitive to velocity changes along an axis parallel to the laser beam, so the kinetic equation has only one velocity variable. Under these assumptions, the function $P$ has the solution $^{6}$

$$
P=\frac{1}{\sqrt{4 \pi D\left(t-t^{\prime}\right)}} \exp \left(\frac{v-v^{\prime}-a\left(t-t^{\prime}\right)}{4 D\left(t-t^{\prime}\right)}\right) .
$$

The probability of optical pumping can then be calculated based on the averaged rate of induced transition for the orbit starting with velocity $v^{\prime}$ :

$$
W\left(t, v^{\prime}, t^{\prime}\right)=\int_{-\infty}^{+\infty} P\left(v, t ; v^{\prime}, t^{\prime}\right) \frac{B I(t)}{c} g\left(v-v_{L}\right) d v .
$$

The function $g$ represents the line shape of the ionic transition and $v_{L}$ is the velocity that an ion must have to Doppler shift the laser light into resonance with the transition [this depends on the laser detuning $\omega-\omega_{0}$ and the optical wave number $\left.k ; v_{L}=\left(\omega-\omega_{0}\right) / k\right]$

$$
g\left(v-v_{L}\right)=\frac{\frac{1}{4} A_{T}^{2}}{k\left(v-v_{L}\right)^{2}+\frac{1}{4} A_{T}^{2}} .
$$

The integral in Eq. (5) is a convolution of a Gaussian and a Lorentzian function (the Voigt function), for example a typical value of $W$ is $10^{6} \mathrm{~s}^{-1}$. Substitution of the result from Eq. (5) into Eq. (1) then results in an equation for the time dependent probability of a particle to remain in the target state. The solution is

$$
p\left(t, v^{\prime}, t^{\prime}\right)=p\left(t^{\prime}\right) \exp \left(-\int_{t^{\prime}}^{t} W\left(t^{\prime \prime} ; v^{\prime}, t^{\prime}\right) d t^{\prime \prime}\right) .
$$

The contribution to the LIF from a given particle is proportional to the optical pumping rate. In a three level system such as in Fig. 1, where one observes photons from the transition $2 \rightarrow 3$, every observable photon comes from an optically pumped ion. Thus the contribution (in photons/s) of an ion initially at velocity $v^{\prime}$ to the LIF signal is

$$
s\left(t, v^{\prime}, t^{\prime}\right)=W\left(t, v^{\prime}, t^{\prime}\right) \frac{A_{23}}{A_{T}} p\left(t, v^{\prime}, t^{\prime}\right) .
$$

The total signal is found by summing over all the initial conditions $v^{\prime}$ according to the initial target state distribution function $f_{0}\left(v^{\prime}, t^{\prime}\right)$ :

$$
S=\int_{-\infty}^{+\infty} s\left(t, v^{\prime}, t^{\prime}\right) f_{0}\left(v^{\prime}, t^{\prime}\right) d v^{\prime}
$$




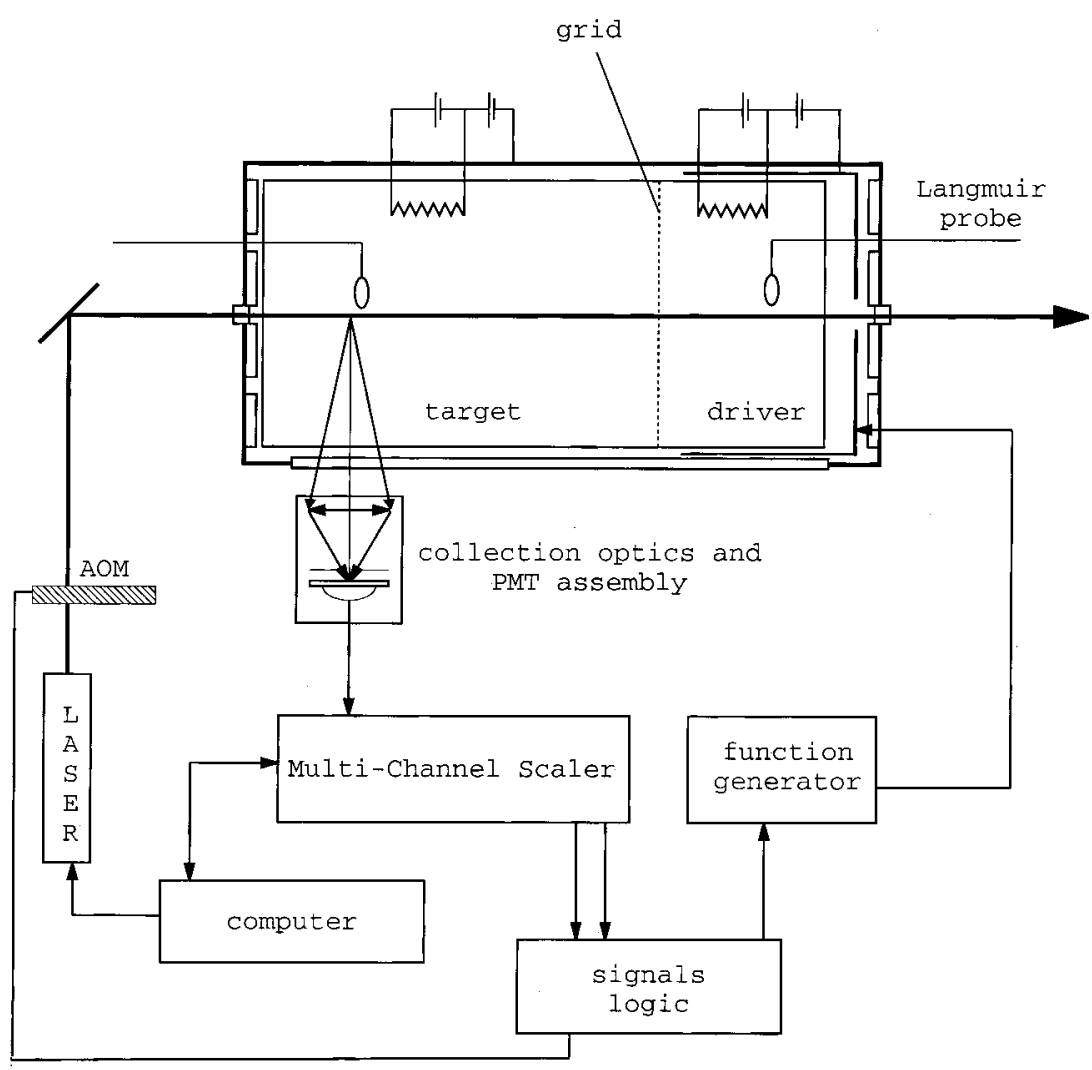

FIG. 2. Experimental setup.

Given the experimental signals, the unknown parameters $a$ and $D$ of Eq. (2) can be determined from fitting to the experimental data. This is done in two steps. First the diffusion coefficient is determined in the quiescent plasma, and then the electric field is determined during an electrostatic shock. ${ }^{7}$

Equation (1) is valid in the limit $A_{T} \gg W \gg r$, where $r$ refers to a typical rate of electronic excitation of the ionic levels involved, which is known only to within an order of magnitude $^{8}$ (see Table I). Electronic excitation produces a background level of fluorescence. When the laser pulse begins, the laser excitation adds to the collisional excitation. When the laser pulse ends the background level of fluorescence requires a brief time to recover due to optical pumping depletion of the target state. Observation of this recovery time indicates the typical electronic collision rate $r$ which we calculate to be $10 \mathrm{kHz}$ for the experiments reported here. Relaxing the inequality $A_{T} \gg W$ results in a finite rise time of the LIF signal (on the order of $1 / A_{T}$ after the start of the laser pulse).

\section{EXPERIMENTAL SETUP}

The experiment is performed in a multipolar Ar II plasma discharge. The $40 \mathrm{~cm}$ diam, $80 \mathrm{~cm}$ long plasma is separated into two chambers by a $250 \mu \mathrm{m}$ step grid of $25 \mu \mathrm{m}$ wires. A schematic of the apparatus is shown in Fig. 2. The driver and target plasma are produced by two similar thermo-electronic discharges with the same parameters shown in Table II. In this double plasma configuration, an ion flux from the driver plasma can be injected in the target by increasing the driver plasma potential.
The laser diagnostic consist of a Coherent 899 ring single-mode continuous-wave dye laser in conjunction with an external acousto-optic modulator. The output of the dye laser, before modulation, has a bandwidth of $0.5 \mathrm{MHz}$, and a center frequency that is electronically tunable. The laser beam is directed through the plasma, perpendicular to the grid. Fluorescence induced by this beam is collected along the radial direction through an optical system, which collimates and filters the light and then is detected by a photomultiplier tube (PMT). The PMT pulses are amplified and counted by a multichannel scaler having a dwell time of 50 ns and a pass length of $38 * 2 * 128=9728$ channels.

As we described above we record both sequences "laser on" and "laser off" to get data for the nonlinear optical tagging. Therefore, the electrostatic shock is sampled in 76 consecutive sequences of laser on and laser off, each sequence having 128 points of $50 \mathrm{~ns}$ (Fig. 3). To obtain a good

TABLE II. Parameters of the experiment. The electron temperature and density are measured by Langmuir probe, the ion temperatures are measured by fitting LIF data to a Maxwellian.

\begin{tabular}{cc}
\hline \hline Parameter & Value \\
\hline$T_{e}$ & $2.5 \mathrm{eV}$ \\
$T_{i}$ & $0.095 \mathrm{eV}$ \\
$n_{e}$ & $5.5 \times 10^{9} \mathrm{~cm}^{-3}$ \\
Pressure & $2 \times 10^{-4} \mathrm{mbar}$ \\
Discharge current & $0.5 \mathrm{~A}$ \\
Discharge potential & $70 \mathrm{~V}$ \\
Duration of the pulse & $156 \mu \mathrm{s}$ \\
Amplitude of the shock & $3.5 \mathrm{~V}$ \\
Offset & $0 \mathrm{~V}$ \\
\hline \hline
\end{tabular}




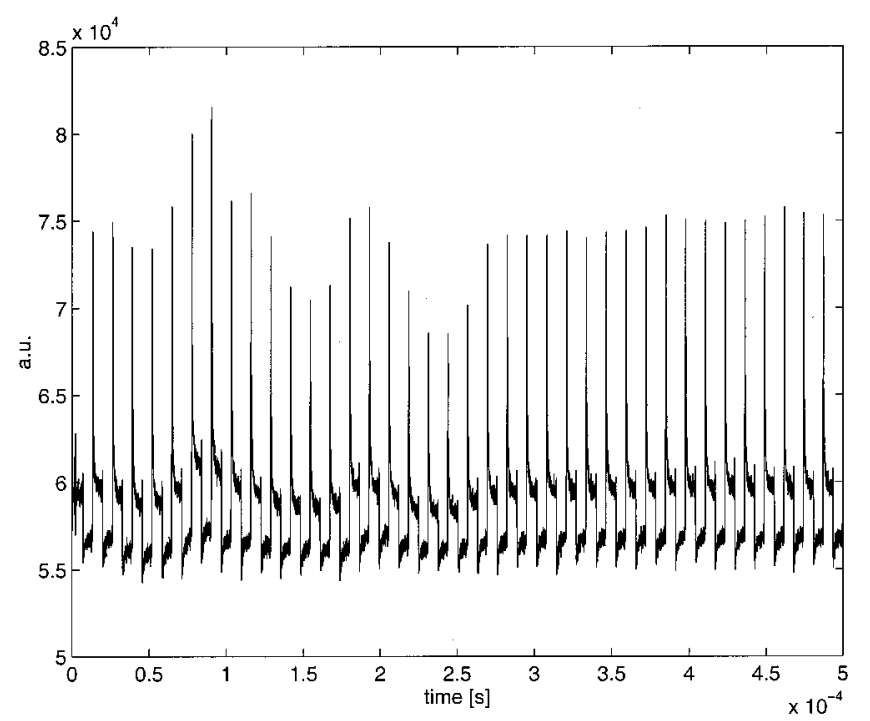

FIG. 3. Fluorescence signal during electrostatic shock.

signal to noise ratio, a cumulative summation is effected.

\section{RESULTS}

The laser frequency is fixed at the maximum of the fluorescence without excitation (corresponding to the mean ion velocity $v_{0}$-in general $\left.v_{0}=0\right)$. The dominant features evident in the data of Fig. 3 are:

(i) a peak in the fluorescence at the start of the laser pulse followed by a decay; and

(ii) a depression after the laser pulse followed by a recovery.

Information is extracted from the data by curve fitting. Starting with the end of the pulse we note that the spontaneous plasma light is depressed after the end of the laser pulse due to depletion of the target state. The recovery is exponential with time constant $\tau$ which is an indication of the repopulation of the target state by electronic collisions, Fig. 4. A value of $\tau=1.5 \times 10^{-6} \mathrm{~s}^{-1}$ is obtained.

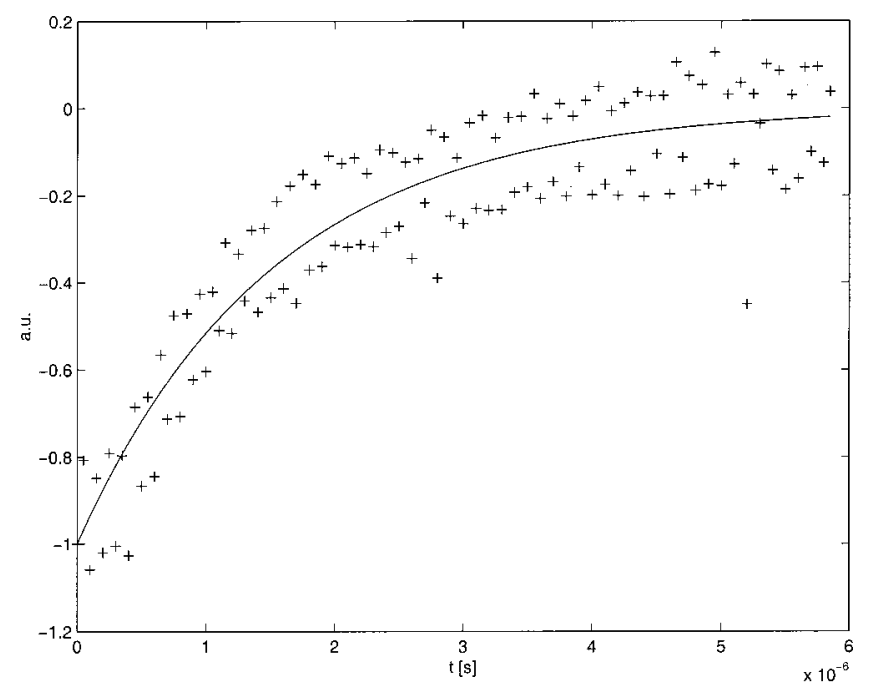

FIG. 4. Evolution of the spontaneous emission when the laser is switched off: (crosses) experimental data, (solid line) the fit; $\tau=1.5 \times 10^{-6} \mu \mathrm{s}$.

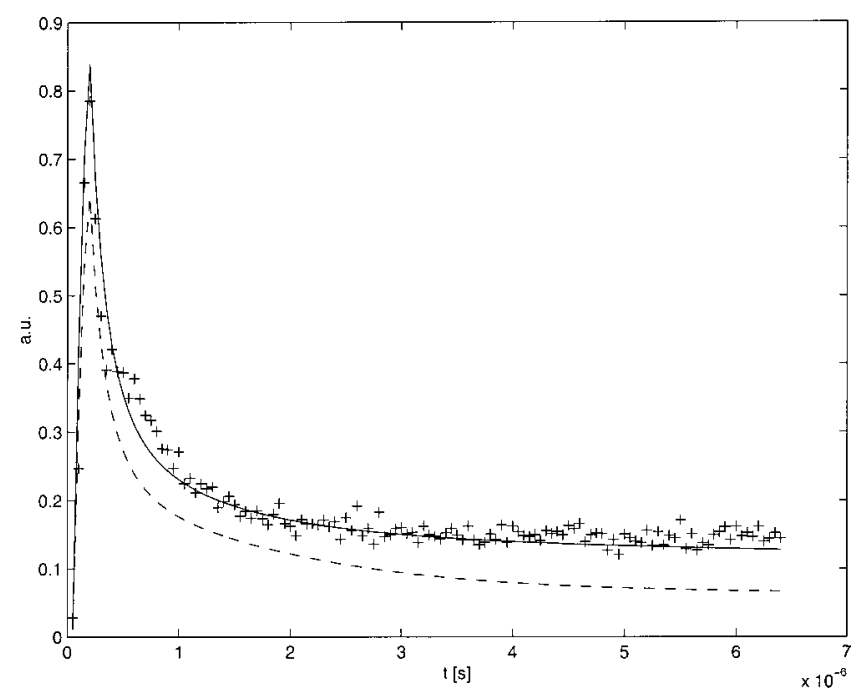

FIG. 5. Electric field effect: (solid line) $E=1.1 \mathrm{~V}$ (best value), (dashed line) $E=-0.6 \mathrm{~V}$, and (crosses) experimental data.

From the fluorescence spike due to LIF in quiescent plasma one can determine the diffusion coefficient $D$ because the decay is nonexponential. ${ }^{3}$ A value of $D=(4.9 \pm 0.8)$ $\times 10^{9} \mathrm{~m}^{2} / \mathrm{s}^{3}$ is obtained which can be compared to the theoretical value of $(5.5 \pm 2.5) \times 10^{9} \mathrm{~m}^{2} / \mathrm{s}^{3}$. The error on the theory value is based on the uncertainty in the plasma density.

Finally, from the fluorescence peaks during the electrostatic shock we introduce nonzero values of the electric field into the model in order to fit the data. The electric field effect in the temporal evolution of the fluorescence is shown in Fig. 5. The effect of the electric field is different but not completely independent of the effect of $D$. Figure 6 shows the resulting electric field. The error bars are determined from the $\chi^{2}$ values of the fits.

As an experimental check of the electric field determination we note that the electric field can be estimated from the dynamics of slow particles (the ones near the peak of the distribution function) if the shock is weak. Such particles will respond linearly to the field of the shock. If we look at

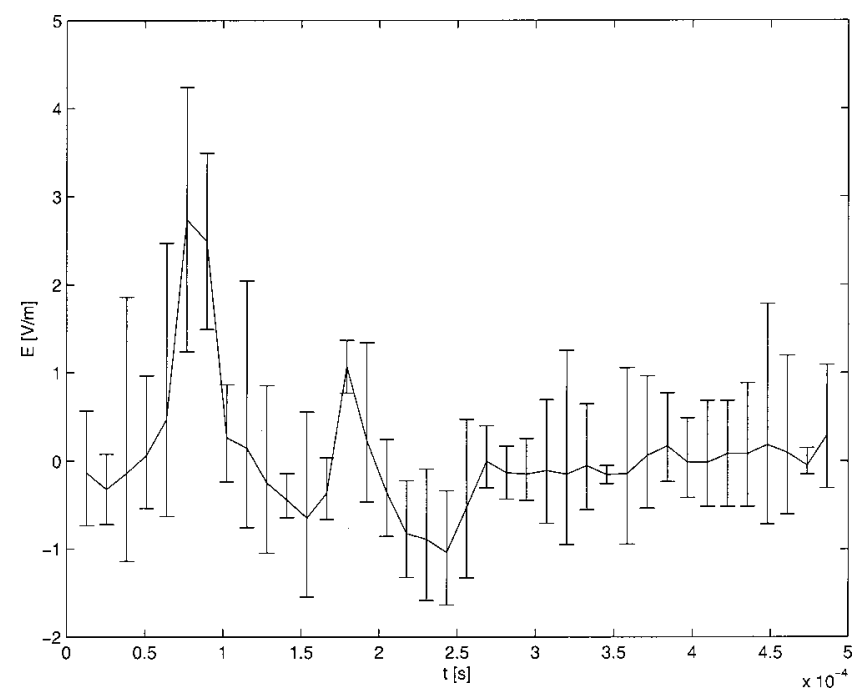

FIG. 6. Electric field during the shock. 


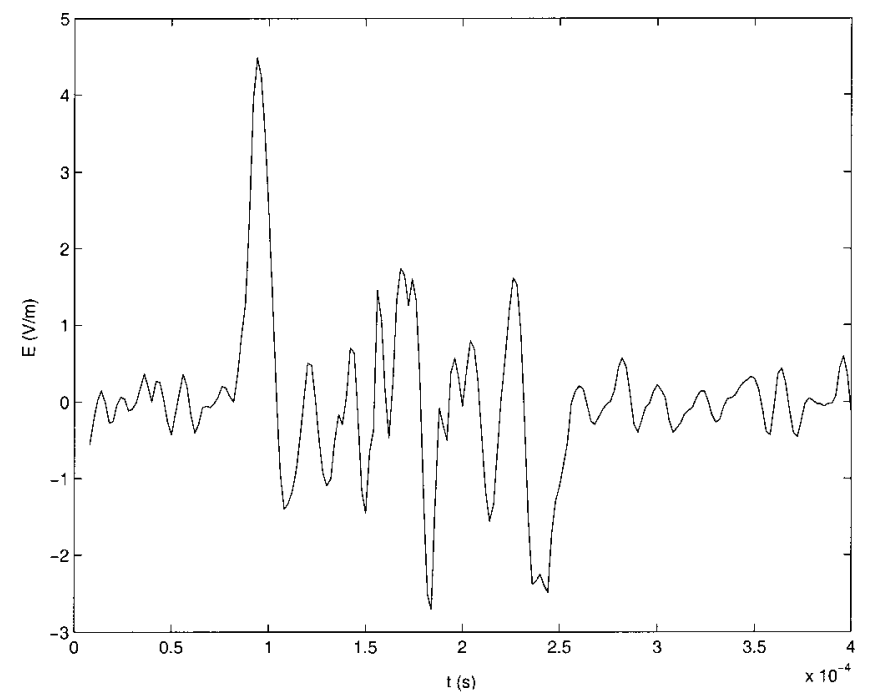

FIG. 7. Estimation of electric field from motion of slow particles.

the Doppler velocity $v_{d}$ corresponding to the peak of the ion distribution, it will satisfy the equation $\frac{1}{2} m v_{d}^{2}+e \phi=$ const. Thus, the electrostatic potential $\phi$ can be determined from the shift in the peak of the distribution $v_{d}$. Figure 7 shows the electric field values obtained by this procedure and they are comparable to the values obtained by the nonlinear optical tagging technique.

\section{DISCUSSION}

We have tested an extension to the nonlinear optical tagging technique by measuring the electric field in an electro- static shock. We note that this technique only requires that the metastable states last long enough to observe the transient optical pumping effect (in this experiment the lifetime need only be a few microseconds). This is much less than the requirement for pump-probe tagging techniques. Because the metastable population has to recover between pulses, there is a limit to the time resolution of the electric field (in this experiment the time resolution was $12.4 \mu \mathrm{s}$ ). If the ion velocities are sufficiently large and the laser beam diameter sufficiently small, then recovery due to physical streaming of ions into the laser beam will occur. In our experiment ions transit the laser beam in about $20 \mu \mathrm{s}$. Experiments to further constrain the possible variations of the diffusion coefficient during the shock are planned.

${ }^{1}$ J. Bowles, R. McWilliams, and N. Rynn, Phys. Plasmas 1, 3814 (1994).

${ }^{2}$ D. N. Hill, S. Fornaca, and M. G. Wickham, Rev. Sci. Instrum. 54, 309 (1983).

${ }^{3}$ J. J. Curry, F. Skiff, M. Sarfaty, and T. N. Good, Phys. Rev. Lett. 74, 1767 (1995).

${ }^{4}$ D. A. Edrich, R. C. McWilliams, and N. S. Wolf, Rev. Sci. Instrum. 67, 2812 (1996).

${ }^{5}$ F. Skiff and J. J. Curry, Rev. Sci. Instrum. 66, 629 (1995).

${ }^{6}$ S. Chandrasekhar, Rev. Mod. Phys. 15, 35 (1943).

${ }^{7}$ G. Bachet, F. Skiff, F. Doveil, and R. A. Stern, Phys. Plasmas 8, 3535 (2001).

${ }^{8}$ H. R. Griem, in Radiative Processes Discharge Plasmas, edited by J. M. Proud and L. H. Luessen, NATO ASI Series B, Vol. 149 (Plenum, New York, 1986), p. 15.

${ }^{9}$ W. B. Thompson and J. Hubbard, Rev. Mod. Phys. 32, 714 (1960). 